\title{
Untersuchungen zur Authentizität von Weinaroma am Beispiel der $\gamma$-Lactone
}

\author{
Ursula Lampe \\ Landesuntersuchungsamt Rheinland-Pfalz, Institut für Lebensmittelchemie und Arzneimittelprüfung, Emy-Roeder-Str. 1, \\ 55129 Mainz, Germany
}

\begin{abstract}
Die Analytik von $\gamma$-Lactonen kann helfen, das Aroma von Weinen unterschiedlicher Rebsorten auf Authentizität zu prüfen. Neben den schon länger bekannten Verfälschungen von Rieslingweinen mit Pfirsicharomen sind so auch Zusätze anderer Fruchtaromen bei vielen weiteren Rebsorten erkennbar.
\end{abstract}

\section{Einführung}

Das Weinaroma wird geprägt durch eine Vielzahl von Aromastoffen, die einerseits aus den Trauben stammen und andererseits im Verlauf der Gärung und der Reifung gebildet werden.

Jede Rebsorte weist ein charakteristisches Aromaprofil auf. Bei Riesling wird das typische Aroma z.B. mit den Begriffen Pfirsich, Aprikose, Apfel, Grapefruit, Rosenblüte und Honig beschrieben. Für die Pfirsichnote im Bouquet von Riesling sind derzeit noch unbekannte Aromastoffe verantwortlich. Der typische Geschmack von Pfirsichen wird dagegen hauptsächlich durch $\gamma$ Decalacton hervorgerufen, aber auch die Homologen $\gamma$ Undecalacton und $\gamma$-Dodecalacton führen $\mathrm{zu}$ diesem Geschmackseindruck.

Alle $\gamma$-Lactone enthalten ein asymmetrisches C-Atom, so dass jeweils zwei enantiomere Formen existieren. $\gamma$ Lactone, die enzymkatalysiert und damit stereoselektiv in der Natur gebildet wurden, zeigen ein stark zum R-Enantiomer verschobenes Enantiomerenverhältnis. Bei chemisch-synthetisch hergestellten $\gamma$-Lactonen liegen demgegenüber beide Enantiomere in etwa gleicher Menge, d.h. als Racemat vor.

Die drei in dieser Arbeit betrachteten $\gamma$-Lactone $(\gamma$ Decalacton, $\gamma$-Undecalacton und $\gamma$-Dodecalacton) treten in authentischen Weinen nicht oder höchstens in geringen Konzentrationen auf, dann jedoch nicht in den oben beschriebenen Enantiomerenverhältnissen. Sie entstehen nicht in nennenswerten Mengen bei der Gärung oder der nachfolgenden Reifung. Auch eine Freisetzung durch Aromaenzyme ist uns nicht bekannt. Lediglich der Einsatz bestimmter Heferindenpräparate kann zur Bildung von $\gamma$ Decalacton in sensorisch relevanten Mengen führen [1]. Die Enantiomeren liegen in diesen Fällen jedoch in einem vom bisher gesagten abweichenden Verhältnis von $\mathrm{R}: \mathrm{S}$ ca. $2: 1$ vor.

Die Erfassung der Enantiomerenverhältnisse der stereoisomeren $\gamma$-Lactone ist daher für die Bewertung der Authentizität eines Weinaromas hinsichtlich einer vorhandenen Pfirsicharomatik von entscheidender Bedeutung.

\section{Material und methoden}

\subsection{Proben}

Untersucht wurden Weine aus dem In- und Ausland sowie einige Aromapräparate.

Für die Kalibrierung wurde ein Modellwein folgender Zusammensetzung als Matrix verwendet: Zucker (Glucose und Fructose je15 g/l), Organische Säuren (Weinsäure, Äpfelsäure und Milchsäure je 1,5 g/l), Gärungsnebenbestandteile (Glycerin 6 g/l, 2,3-Butandiol $1 \mathrm{~g} / \mathrm{l}, 2$-Phenylethanol $40 \mathrm{mg} / \mathrm{l})$ sowie Ethanol (12\%vol), eingestellt auf $\mathrm{pH}=3,5 \mathrm{mit}$ Kalilauge, wodurch ein Kaliumgehalt von ca. $1 \mathrm{~g} / \mathrm{l}$ resultiert. Der Modellwein wurde mit steigenden Mengen an $\gamma$-Lactonen $(\gamma$ Decalacton, $\gamma$-Undecalacton und $\gamma$-Dodecalacton) im Konzentrationsbereich von ca. 0,5 - $25 \mu \mathrm{g} / \mathrm{l}$ je Enantiomer dotiert und dann in gleicher Weise wie die Weinproben extrahiert.

\subsection{Probenvorbereitung}

Die $\gamma$-Lactone wurden durch eine modifizierte Flüssigextbreakraktion mit Kaltron nach Rapp [2] aus der Weinmatrix abgetrennt: $20 \mathrm{ml}$ Wein wurden mit dem internem Standard $\varepsilon$-Decalacton gemischt und nach Zugabe von Kochsalz bis fast zur Sättigung mit $400 \mu \mathrm{l}$ Kaltron für 15 min geschüttelt. Zur besseren Phasentrennung wurde zunächst gekühlt und dann zentrifugiert. Die untere, organische Phase wurde zur Messung eingesetzt.

\subsection{Messung mittels Gaschromatographie I Massenspektrometrie (GC/MS)}

Die Trennung der $\gamma$-Lactone erfolgte durch chirospezifische Gaschromatographie an einer chiralen Trennsäule (BGB-174, belegt mit B-Cyclodextrin in einer 1701Phase). Die Messung erfolgte an einem GC/MS-System von Thermo Scientific (Trace GC Ultra, DSQ II). Zur Detektion wurde der SIM-Scan-Modus eingesetzt: m/z 85 und m/z $128 \mathrm{im} \mathrm{SIM-Modus} \mathrm{sowie} \mathrm{m/z} 50$ bis m/z 130 
Tabelle 1. Beispiele für erkannte Aromaverfälschungen bei Weinen verschiedener Rebsorten.

\begin{tabular}{|c|c|c|c|}
\hline Rebsorte & Sensorik & $\gamma$-Decalacton & $\gamma$-Undecalacton \\
\hline Kadarka & $\begin{array}{l}\text { Kräuter, } \\
\text { Sangria }\end{array}$ & $\begin{array}{l}\mathrm{R}+\mathrm{S}=15,3 \mu \mathrm{g} / \mathrm{l} \\
\mathrm{R}: \mathrm{S}=92: 8\end{array}$ & $\begin{array}{l}\mathrm{R}+\mathrm{S}=1,3 \mu \mathrm{g} / \mathrm{l} \\
\mathrm{R}: \mathrm{S}=54: 46\end{array}$ \\
\hline Portugieser & Erdbeer & $\begin{array}{l}\mathrm{R}+\mathrm{S}=17,3 \mu \mathrm{g} / \mathrm{l} \\
\mathrm{R}: \mathrm{S}=50: 50\end{array}$ & - \\
\hline $\begin{array}{l}\text { Sauvignon } \\
\text { Blanc }\end{array}$ & $\begin{array}{l}\text { Citrus, } \\
\text { Sangria }\end{array}$ & - & $\begin{array}{l}\mathrm{R}+\mathrm{S}=20,6 \mu \mathrm{g} / 1 \\
\mathrm{R}: \mathrm{S}=50: 50\end{array}$ \\
\hline Rivaner & Pfirsich & $\begin{array}{l}\mathrm{R}+\mathrm{S}=10,5 \mu \mathrm{g} / \mathrm{l} \\
\mathrm{R}: \mathrm{S}=52: 48\end{array}$ & $\begin{array}{l}\mathrm{R}+\mathrm{S}=5,3 \mu \mathrm{g} / \mathrm{l} \\
\mathrm{R}: \mathrm{S}=50: 50\end{array}$ \\
\hline Chardonnay & Pfirsich & - & $\begin{array}{l}\mathrm{R}+\mathrm{S}=32,2 \mu \mathrm{g} / \mathrm{l} \\
\mathrm{R}: \mathrm{S}=50: 50\end{array}$ \\
\hline
\end{tabular}

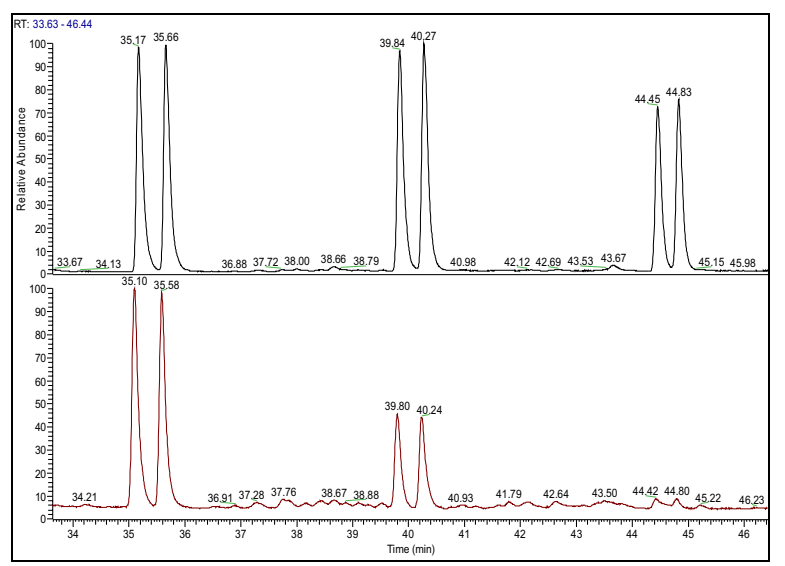

Abb 1. GC/MS-Chromatogramme, SIM-Modus, m/z 85 oben: Kalibrierstandard gemäß 2.1 (R- und S- $\gamma$-Decalacton: 35,17 min und 35,66 min; R- und S- $\gamma$-Undecalacton: $39,84 \mathrm{~min}$ und 40,27 min; R- und S- $\gamma$-Dodecalacton: 44,45 min und 44,83 min) unten: Riesling mit Zusatz eines Pfirsich-Aromas, bestehend aus $\gamma$-Decalacton und $\gamma$-Undecalacton, jeweils racemisch (chemisch-synthetische Herstellung).

im Scan-Modus. Die Quantifizierung erfolgte anhand der SIM-Daten, die full-scan-Massenspektren wurden zur qualitativen Absicherung herangezogen.

\section{Ergebnisse und diskussion}

\subsection{Weine der Rebsorte Riesling}

Die Aromabeschreibungen von Riesling-Weinen enthalten stets als erwünschten Geschmackseindruck einen "Pfirsich-Ton", der mit kellertechnischen Maßnahmen allein nicht immer erreicht werden kann. Daher ist offensichtlich die Versuchung groß, das Bouquet durch Zugabe von Pfirsicharomen zu verbessern. Die beschriebene Methode zur stereoselektiven Messung der $\gamma$-Lactone ermöglicht es, eine solche Veränderung des Weinaromas sicher zu erkennen.

Handelsübliche Aromapräparate vom Typ "Pfirsich" z.B. für die Likörproduktion können die drei genannten $\gamma$-Lactone entweder einzeln oder in jeder Kombination, racemisch oder in einem natürlichen Enantiomerenverhältnis enthalten. Dementsprechend zeigen all diese

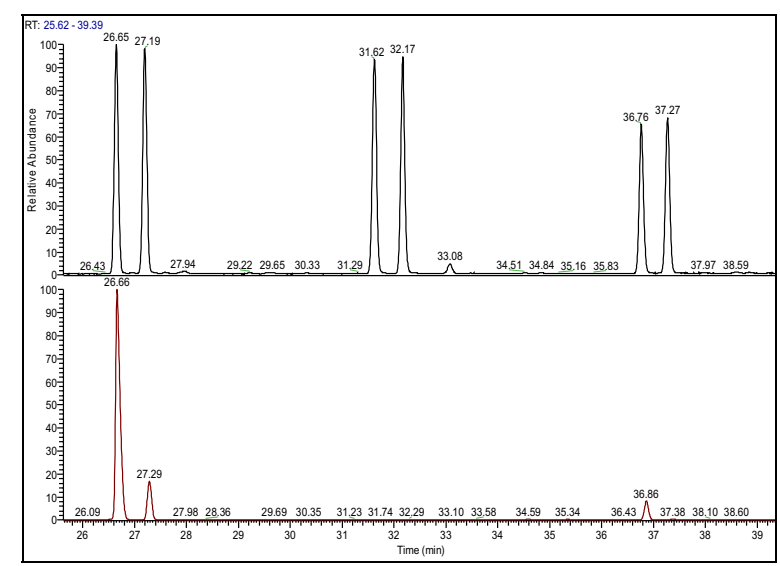

Abb 2. GC/MS-Chromatogramme, SIM-Modus, m/z 85 oben: Kalibrierstandard gemäß 2.1 (R- und S- $\gamma$-Decalacton: 26,65 min und 27,19 min; R- und S- $\gamma$-Undecalacton: $31,62 \mathrm{~min}$ und $32,17 \mathrm{~min}$; R- und S- $\gamma$-Dodecalacton: 36,76 min und 37,27 min) unten: Riesling mit Zusatz eines Pfirsich-Aromas, bestehend aus $\gamma$-Decalacton und $\gamma$-Dodecalacton, jeweils R:S $>80: 20$ (Aromaextrakt aus Pfirsichen).

Kombinationen ein verändertes Weinaroma an, wenn sie in Weinen so angetroffen werden, z.B.:

- zwei oder alle drei genannten $\gamma$-Lactone sind racemisch in unterschiedlichen Mengen enthalten (Abb. 1.),

- ausschließlich $\gamma$-Decalacton oder $\gamma$-Undecalacton ist racemisch enthalten,

- vorwiegend R- $\gamma$-Decalacton ist neben wenig S- $\gamma$ Decalacton enthalten (R:S > 80:20), ggf. mit geringem Anteil an R- $\gamma$-Dodecalacton (Abb. 2.),

- nur R- $\gamma$-Decalacton und racemisches $\gamma$-Undecalacton sind enthalten.

\subsection{Weine anderer Rebsorten}

Mit Hilfe der beschriebenen Lactonanalytik lassen sich aber nicht nur Zusätze von Pfirsicharomen in Rieslingweinen erkennen. Bei anderen Rebsorten werden vielfältige weitere fruchtige Geschmacksnoten erwartet, für die ebenfalls passende Aromapräparate im Handel angeboten werden. In vielen Fruchtaromen werden neben den Hauptaromastoffen für die jeweilige Geschmacksrichtung oft auch $\gamma$-Lactone in deutlich geringeren Anteilen zur Erzielung einer unspezifischen Fruchtnote eingesetzt, wie Beispiele aus der Fachliteratur zeigen $[3,4]$.

Aufgrund dieser Zusammenhänge konnten auch in Weinen anderer Rebsorten bereits einige Aromaverfälschungen erkannt werden (Tabelle 1):

- Ein Rotwein der Rebsorte Kadarka wurde in der Sensorik als "untypisch kräuterartig mit Sangriageschmack" beschrieben. Die Lactonanalytik zeigte ein nicht weintypisches Aromaspektrum: viel $\gamma$-Decalacton mit stark verschobenem Enantiomerenverhältnis $(\mathrm{R}: \mathrm{S}=$ 92:8) sowie wenig $\gamma$-Undecalacton racemisch.

- Des weiteren fiel ein Rotwein der Rebsorte Portugieser sensorisch durch einen erkennbaren Erdbeerton auf. 
Der Wein enthielt $\gamma$-Decalacton als Racemat und war folglich verfälscht. Nachforschungen beim Hersteller ergaben schließlich, dass dem Wein ein Erdbeersirup aus dem Einzelhandel zugesetzt worden war.

- Ein Weißwein der Rebsorte Sauvignon Blanc, der sensorisch den Eindruck von Citrus und Sangria aufwies, enthielt racemisches $\gamma$-Undecalacton.

- Zwei Weißweine der Rebsorte Rivaner bzw. Chardonnay mit erkennbarem Pfirsichton wiesen beide $\gamma$-Lactone als Racemat auf.

\section{Literatur}

[1] U. Arzberger, Die Säulen der Weinüberwachung (Bundesausschuss für Weinforschung, 2010)

[2] A. Rapp, I. Yavas, H. Hastrich, Deut. Lebensm.Rundsch. 90, 171 (1994)

[3] E. Ziegler, Die natürlichen und künstlichen Aromen (Dr. Alfred Hüthig Verlag GmbH Heidelberg, 1982)

[4] G. Fenaroli, Fenaroli's handbook of flavor ingredients, Vol. II, 3rd edition (CRC Press, Inc., 1995) 